Check for updates

Cite this: RSC Adv., 2017, 7, 45676

\title{
Far-red fluorescent carbon nano-onions as a biocompatible platform for cellular imaging $\dagger$
}

\author{
Stefania Lettieri, ${ }^{a}$ Adalberto Camisasca, ${ }^{\text {ab }}$ Marta d'Amora, ${ }^{c}$ Alberto Diaspro, ${ }^{\text {cde }}$ \\ Takashi Uchida, ${ }^{f}$ Yoshikata Nakajima, ${ }^{f}$ Keiichi Yanagisawa, ${ }^{f}$ Toru Maekawa $^{f}$ \\ and Silvia Giordani (iD *ag
}

A new generation of fluorescent carbon nano-onions with enhanced solubility in biological media and bright photoluminescence is reported. The nano-onions functionalized with a water soluble boron dipyrromethene dye emit in the far red spectrum with a high quantum yield $\left(\Phi_{\mathrm{F}}\right)$ and are suitable for high resolution imaging. The nanoparticles are characterized by a variety of different analytical techniques such as thermogravimetric analysis, dynamic light scattering, zeta potential, electron microscopy, Raman, $X$-ray photoelectron and fluorescence spectroscopies. They are easily internalized by human breast cancer cells (MCF-7) without any significant toxic effects. Moreover, confocal imaging studies show they exhibit a high fluorescence intensity and are localized in the lysosomes at a very low concentration. Our findings confirm the excellent potentialities of these functionalized carbon nanomaterials as biocompatible platform for high resolution biological imaging

Received 25th August 2017

Accepted 15th September 2017

DOI: 10.1039/c7ra09442f

rsc.li/rsc-advances

and far-red region, where tissue exhibit minimal absorbance. ${ }^{11}$

\section{Introduction}

Carbon Nano Materials (CNMs) such as carbon nanotubes, ${ }^{1-3}$ fullerene, ${ }^{4}$ carbon dots $^{5}$ and carbon nano-onions, ${ }^{6}$ are an attractive platform for biomedical applications due to their biocompatibility, small size (which ranges between $1 \mathrm{~nm}$ to 100 $\mathrm{nm}$ ) and large surface area. The development of fluorescent carriers as optical imaging tools became in the past years relevant, both for the localization of specialized nanoparticles in cells, tissue and organs, and in diagnostics. ${ }^{7,8}$ Furthermore, imaging as a non-invasive technique, which involves the use of visible, and infrared light decreasing the exposure of the patient to harmful radiation, ${ }^{9}$ can be applied for live study event at a molecular level and as diagnostic tool for the prevention and treatment of cancer and other diseases. ${ }^{\mathbf{1 0}}$ One of the strategies to enhance bioimaging relies on the development of imaging probes emitting in the biologically relevant near-infra red (NIR)

${ }^{a}$ Nano Carbon Materials, Istituto Italiano di Tecnologia (IIT), via Morego 30, 16163, Genoa, Italy.E-mail: silvia.giordani@iit.it

${ }^{b}$ Department of Chemistry, University of Genoa, Via Dodecaneso 31, Genoa, 16145, Italy

'Optical Nanoscopy, Nanophysics, Istituto Italiano di Tecnologia (IIT), via Morego 30, 16163, Genoa, Italy

${ }^{d}$ NIC@IIT, Istituto Italiano di Tecnologia (IIT), Via Morego 30, Genoa, 16163, Italy ${ }^{e}$ Department of Physics, University of Genoa, Via Dodecaneso 33, Genoa, 16145, Italy ${ }^{f}$ Bio-Nano Electronics Research Centre, Toyo University 2100, Kujirai, Kawagoe, Saitama 350-8585, Japan

${ }^{g}$ Department of Chemistry, University of Turin, via Giuria 7, 10125, Turin, Italy $\dagger$ Electronic supplementary information (ESI) available: Material and methods, various synthetic procedures, additional spectroscopy and biological data. See DOI: $10.1039 / \mathrm{c} 7 \mathrm{ra09442f}$
Thus there is the need for the developments of new organic dye molecules emitting in the far-red and NIR region ${ }^{12}$ to decrease the tissue auto-fluorescence allowing a high resolution imaging and a deeper tissue penetration. Multi-shell fullerenes, known as carbon nano-onions (CNOs), ${ }^{\mathbf{1 3}, \mathbf{1 4}}$ are an attractive class of CNMs for imaging, diagnostic and therapeutic applications, due to their small size, spherical shape ${ }^{15}$ and low toxicity. After their discovery by Iijima in $1980,{ }^{16}$ Ugarte reported the in situ transformation of amorphous carbon into onion-like graphitic nanoparticles. ${ }^{13}$ Different production methods of CNOs have been reported so far, including arc discharge, ${ }^{17}$ pyrolysis, ${ }^{18,19}$ chemical vapour deposition (CVD) ${ }^{20}$ and thermal annealing of detonation nano-diamonds (d-NDs) in vacuum ${ }^{21}$ or under inert atmosphere..$^{22,23}$ In general, CNMs display poor solubility in aqueous solvents where they are prone to aggregation due to hydrophobic interactions. However, this can be addressed through surface functionalization by covalent ${ }^{24-26}$ or noncovalent methods. ${ }^{26-30}$ In particular, several functionalization pathways have been reported where CNOs were chemically functionalized on their large surface area, covalently through chemical reaction directly with the $\mathrm{sp}^{2}$ carbon atoms present on the material surface, ${ }^{6}$ and through adsorption of organic molecules by $\pi-\pi$ stacking between the outer graphitic layer of the CNO and the aromatic moiety of a bioactive molecule. ${ }^{31}$ These well-establish surface modifications (e.g. oxidation, ${ }^{32,33}$ cycloaddition, ${ }^{34}$ fluorination, ${ }^{35}$ radical addition of diazonium compounds ${ }^{36}$ ), allow chemist to create specialized nanoparticles soluble in aqueous environments and biocompatible. Our recent reports showed that fluorescently labelled CNOs 
exhibit weak inflammatory potential, a low cytotoxicity, ${ }^{33}$ they are readily internalized by cancer cells and accumulate in the lysosomes. ${ }^{37,38}$ Furthermore, our in vivo studies performed on zebrafish (Danio Rerio) during development have demonstrated their biocompatibility. ${ }^{39}$ However, the potential of CNMs for biological application can be further enhanced by increasing their solubility in water-based medium obtaining small specialized nanoparticles with a narrow size distribution. Here, we report a new generation of fluorescent far-red carbon nanoonions with a great dispersibility in biological media for biomedical applications. The quaternary ammonium salt derivative of a $\pi$-extended distyryl-substituted boron dipyrromethene dye $\mathrm{40,41}^{\mathbf{4}}$ leads to the synthesis of a water soluble BODIPY molecule. The latter is designed to improve the solubility of CNO in cellular medium and to improve the emitting properties of the CNO upon cell internalisation. The CNOs grafted with the water-soluble fluorophore show bright fluorescence in both organic solvents and cell medium, proving their suitability as bioimaging probes. We demonstrate the successful functionalization of the CNOs using a variety of different analytical techniques such as thermogravimetric analysis (TGA), dynamic light scattering (DLS), zeta potential, electron microscopy, Raman, X-ray photoelectron and fluorescence spectroscopies. Similarly, in vitro experiments in MCF-7 cells show an excellent cellular uptake, low toxicity, and a bright photoluminescence at the confocal microscope of those fluorescent probes. Our finding pave the way for the development of high resolution biological imaging platform.

\section{Results and discussion}

\section{Synthetic procedures}

The synthetic procedures are shown in Schemes 1 and 2 . BODIPY 1 was synthetized following a previously reported procedure. $^{12}$ The condensation with dimethylaminobenzaldehyde led to the NIR-BODIPY derivative 2 . The water soluble BODIPY 3 was obtained through the quaternarization of 2. Pristine CNOs (p-CNOs), synthetized by thermal annealing of d-NDs, were oxidized using a $3 \mathrm{M}$ solution of nitric acid under reflux conditions.

The oxidation was performed directly on the $\mathrm{sp}^{2}$ carbon present on the p-CNOs surface, leading to the creation of hybridized $\mathrm{sp}^{3}$ carbon atoms. The oxidized CNOs (oxi-CNOs) were grafted with BODIPY 3 molecules through an esterification

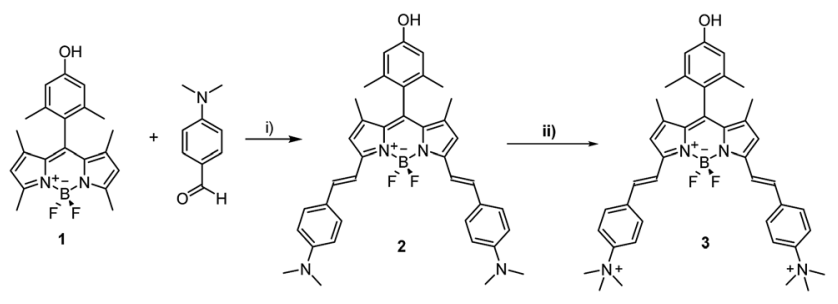

Scheme 1 Synthesis of BODIPY derivatives 2 and 3. (i) Toluene, piperidine, glacial acetic acid, $\mathrm{Mg}\left(\mathrm{ClO}_{4}\right)_{2}$, Dean-Stark condenser; (ii) acetone, $\mathrm{CH}_{3}$.

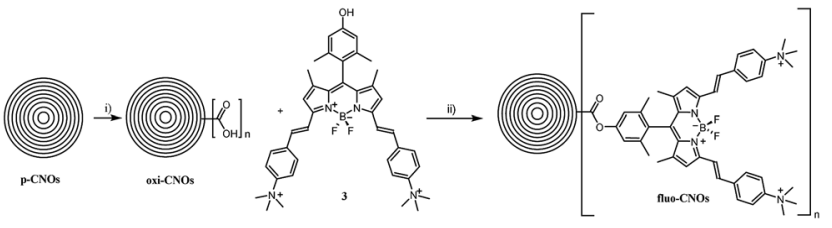

Scheme 2 Functionalization of oxi-CNOs with BODIPY 3. (i) Nitric acid, reflux, 48 hours; (ii) EDC, NHS, DMAP, 3, dry DMF, $\mathrm{N}_{2}, \mathrm{RT}$, 20 hours

procedure using 1-ethyl-3-(3-dimethylaminopropyl) carbodiimide (EDC) as the coupling agent (Scheme 2) to obtain fluoCNOs. The detailed synthetic procedures are reported in the ESI. $\dagger$

\section{Characterization of CNO}

Oxi- and fluo-CNOs were characterized by several techniques (Raman, TGA, and XPS) to prove the successful surface functionalization of CNOs.

The Raman spectra of p-, oxi- and fluo-CNOs showed a D-band $\left(1320 \mathrm{~cm}^{-1}\right)$ and a G-band $\left(1580 \mathrm{~cm}^{-1}\right)$ typical of CNO. ${ }^{37}$ The D-band at $1320 \mathrm{~cm}^{-1}$ refers to the defects present on the outer graphitic layer and is due to the presence of $\mathrm{sp}^{3}$ hybridazed carbons. The G-band at around $1580 \mathrm{~cm}^{-1}$ corresponds to the $\mathrm{E}_{2 \mathrm{~g}}$ mode of $\mathrm{sp}^{2}$-hybridized carbon frameworks. The D/G ratio increased from p-CNOs to oxi-CNOs due to the introduction of defects on the CNOs outer layer by the oxidation process (Fig. 1a). No difference was observed between oxi-CNOs and fluo-CNOs as the coupling reaction didn't introduce new functionalities directly on the hybridized $\mathrm{sp}^{2}$ carbon atoms. TGA analyses, performed in air, confirmed the successful functionalization of the CNOs surface (Fig. 1b). The decomposition temperature decreased from $686{ }^{\circ} \mathrm{C}$ (p-CNOs) to $668{ }^{\circ} \mathrm{C}$ (oxi-CNOs). From the TGA weight loss at $450{ }^{\circ} \mathrm{C}$, we estimated approx 120 carboxylic groups per CNO in the oxi-CNOs and around 5 dye molecules per CNO in the fluo-CNOs.

XPS survey spectrum of the functionalized CNOs was acquired, thus measuring the elemental composition of each CNO sample. In Table 1 are reported in detail the percentage of each element per CNO. As expected, the oxygen content increased upon oxidation, which we attribute to the introduction of - $\mathrm{COOH}$ groups in the oxi-CNOs.

The XPS survey spectrum of fluo-CNOs shows a sample composition of carbon $(90.1 \%)$, oxygen $(7.1 \%)$, nitrogen $(1.9 \%)$ and fluorine $(0.9 \%)$ confirming the presence of BODIPY 3 molecules on the CNO surface. Indeed the nitrogen and fluorine content are in a ratio of $2: 1$ as in the BODIPY 3 (four nitrogen and two fluorine atoms are present per dye molecule). In order to investigate the chemical state of each element, highresolution C 1s XPS spectra of CNO derivatives (Fig. 1c) were acquired. A fitting was performed for every peak and the different contributes were assigned after the deconvolution. The C 1s spectrum of p-CNOs is composed of a main peak at $284.5 \mathrm{eV}$ corresponding to the presence of $\mathrm{sp}^{2}$ carbon atoms and four other peaks referring to the presence of $\mathrm{sp}^{3}$ carbon atoms (285.4 eV), oxygen-bonded carbon $(\mathrm{C}-\mathrm{O}$ and $\mathrm{C}=\mathrm{O}$ at $286.5 \mathrm{eV}$ 

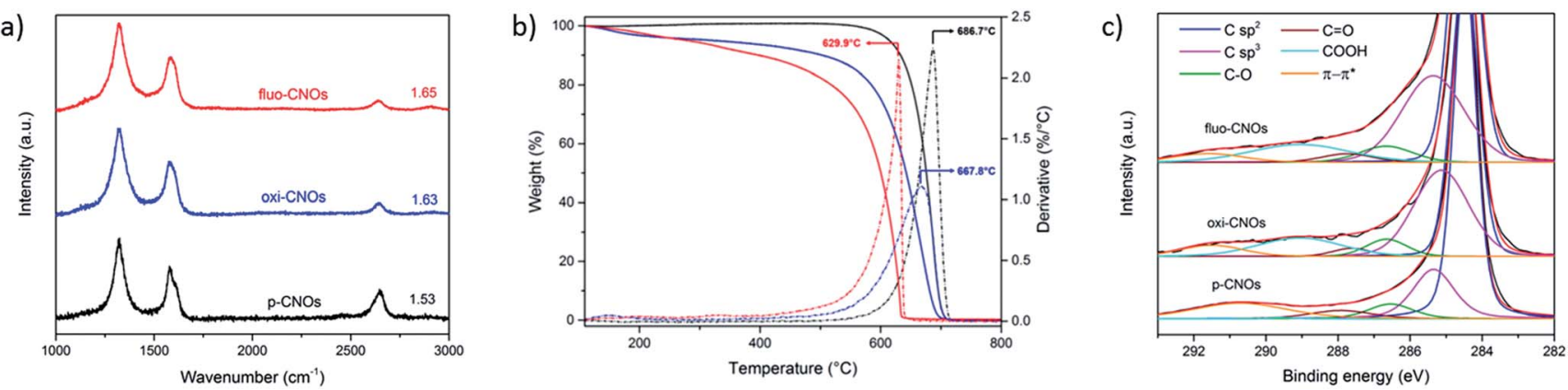

Fig. 1 Raman, TGA and XPS of the CNOs. (a) The Raman spectra are normalized for the G-band at $1580 \mathrm{~cm}^{-1}$ and the ratios of the D-band to the G-band intensities are indicated; (b) thermogravimetric analysis (solid lines) and the corresponding weight loss derivatives (dotted lines) of $\mathrm{p}$-CNOs (black), oxi-CNOs (blue) and fluo-CNOs (red). All experiments are run in air with a temperature rate of $10^{\circ} \mathrm{C} \mathrm{min}{ }^{-1}$; (c) XPS C $1 \mathrm{~s} \mathrm{spectra}$ of $\mathrm{p}-$, oxi- and fluo-CNOs, including peak-fitting analyses, showing the presence of the peak at about $289 \mathrm{eV}$ corresponding to the carboxylic acid functionalities.

and $287.9 \mathrm{eV}$ respectively) species and $\pi-\pi^{*}$ interactions $(290.7 \mathrm{eV})$. The C $1 \mathrm{~s}$ spectrum of oxi-CNOs is composed by the same peaks of the pristine type and a new peak corresponding to the $-\mathrm{COOH}$ species $(289 \mathrm{eV})$, thus supporting the successful introduction of carboxylic acid groups onto the surface of CNOs.

No differences were observed in the $\mathrm{C} 1 \mathrm{~s}$ spectrum of CNO after coupling with the dye, as no changes in the carbon atoms chemical state were present. The chemical state and the area (\%) for every peaks were reported in ESI (Table S1 $\uparrow$ ).

Dynamic light scattering (DLS) measurements are carried out to determine the hydrodynamic radius of the dispersed CNOs (Table 2, Fig. S12 and 13 in the ESI $†$ ). DLS experiments were performed on CNOs samples dispersed in PBS $0.01 \mathrm{M}$. The dispersions of oxi-CNOs or fluo-CNOs were prepared at an initial concentration of $1 \mathrm{mg} \mathrm{mL}{ }^{-1}$ by sonication of the sample for 30 minutes in water followed by dilution of the samples in the final solvent to achieve final concentrations of 5, 10 and $20 \mu \mathrm{g} \mathrm{mL} \mathrm{m}^{-1}$. Oxi-CNOs revealed an effective hydrodynamic diameter in PBS of $274 \pm 15.7 \mathrm{~nm}$, while the fluo-CNOs exhibited an average diameter of $295 \pm 17.4 \mathrm{~nm}$. Therefore, the functionalization of the CNOs with BODIPY 3 does not affect the stability and dispersibility of the latter in aqueous medium, due to the solubility of the dye in water.

HRTEM images (Fig. 2) clearly show the concentric graphitic layers structure of CNOs, ${ }^{14}$ with an inter-graphitic layer distance of $3.4 \AA$. No changes were observed on the CNOs structure following surface functionalization.

The spectroscopic properties of the dye 3 and of the fluoCNOs are shown in Table 3. Upon photoexcitation of $550 \mathrm{~nm}$ of both BODIPY 3 and fluo-CNOs in DMSO, an emission band centered at $634 \mathrm{~nm}$ was observed as shown in Fig. 3. The inset of

Table 1 Elemental composition of $\mathrm{p}$-, oxi- and fluo-CNOs obtained by XPS measurements

\begin{tabular}{lllll}
\hline Sample & C (\%) & O (\%) & N (\%) & F (\%) \\
\hline p-CNOs & 99.0 & 1.0 & - & - \\
oxi-CNOs & 90.7 & 9.3 & - & - \\
fluo-CNOs & 90.1 & 7.1 & 1.9 & 0.9
\end{tabular}

Fig. 3 shows the emission spectra of the BODIPY 3 alone (red line) and fluo-CNOs (back line) which demonstrate the successful functionalization of the CNOs with the dye molecule.

In water, the absorption and emission of the dye molecule were slightly blue shifted (hypsochromic effect) with a $\lambda_{\text {Abs }}$ at $616 \mathrm{~nm}$, and a $\lambda_{\mathrm{Em}}$ at $627 \mathrm{~nm}$. BODIPY 3 exhibited a high quantum yield $\left(\Phi_{\mathrm{F}}\right)$ of 0.8 in DMSO and a molar extinction coefficient $(\varepsilon)$ of $88.28 \times 103 \mathrm{M}^{-1} \mathrm{~cm}^{-1}$ at a wavelength of $627 \mathrm{~nm}$. Remarkably, the herein reported fluo-CNOs maintained a high quantum yield of 0.4 in DMSO. The emission intensity reported in the literature for dyes linked to the surface of CNMs were much lower (less than a third, compare to the intensity of the dye alone). ${ }^{42}$ Moreover the emission spectrum of fluo-CNOs was recorded in cell medium (DMEM) to see if the photoluminescence of the fluorescent CNOs was preserved in aqueous medium proving their suitability in bio applications (Fig. S7 in the ESI $\dagger$ ). The fluo-CNOs exhibited a bright fluorescence centered at $632 \mathrm{~nm}$ in DMEM. All absorption and emission spectra of the BODIPY 3 and fluo-CNOs in the different media are reported in the ESI. $\dagger$

\section{Cytotoxicity studies}

Previous in vitro and in vivo studies of functionalized CNOs demonstrate their low cytotoxicity, ${ }^{33}$ and a good biocompatibility in a small vertebrate model, ${ }^{39}$ suggesting that CNO is a biocompatible and safe nanomaterial. The herein reported

Table 2 Effective hydrodynamic diameter obtained from dynamic light scattering (DLS) measurements and zeta-potential of oxi-CNOs and fluo-CNOs at a concentration of $5 \mu \mathrm{g} \mathrm{mL}^{-1}$

\begin{tabular}{lll}
\hline Sample & $\begin{array}{l}\text { Effective hydrodynamic } \\
\text { diameter }(\mathrm{nm})\end{array}$ & $\begin{array}{l}\text { Zeta potential } \\
(\mathrm{mV})\end{array}$ \\
\cline { 2 - 3 } & $0.01 \mathrm{M} \mathrm{PBS}$ & $\begin{array}{l}\text { Phosphate } \\
\text { buffer }\end{array}$ \\
\hline $\begin{array}{lll}\text { oxi-CNOs } \\
\text { fluo-CNOs }\end{array}$ & $274 \pm 16$ & $-45 \pm 5$ \\
& $295 \pm 17$ & $-34 \pm 4$
\end{tabular}



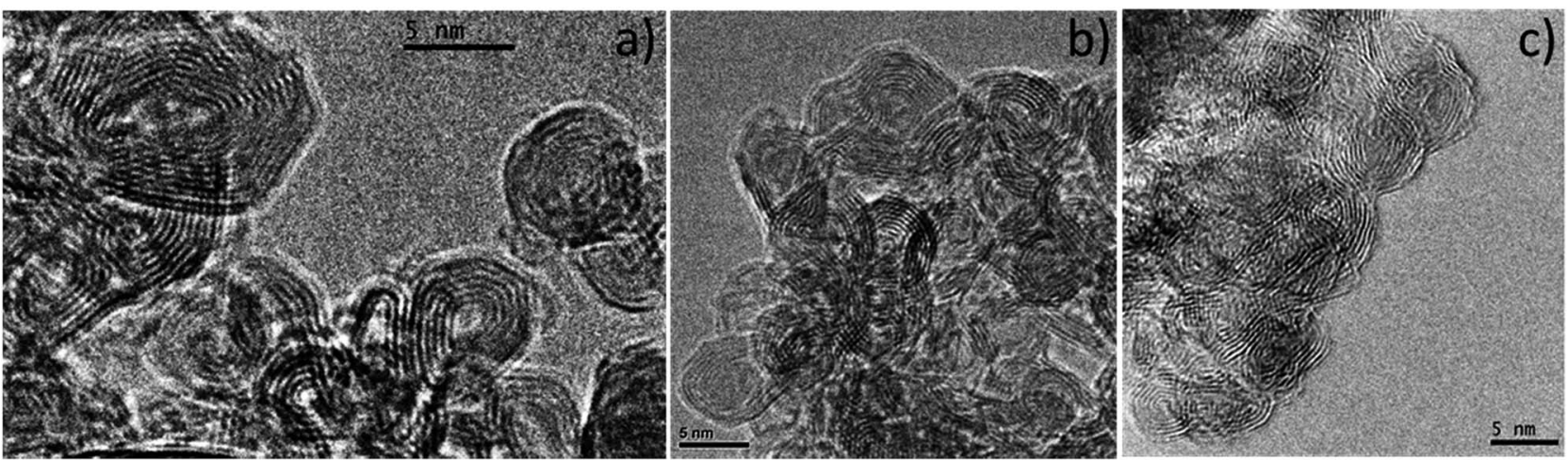

Fig. 2 Representative high-resolution TEM images of p-CNOs (a), oxi-CNOs (b) and fluo-CNOs (c).

Table 3 Photophysical data for BODIPY 3 and fluo-CNOs. $\lambda_{\text {Abs }}$ max: absorption maximum; $\lambda_{\mathrm{em}}$ max: Emission maximum; $\Phi_{\mathrm{F}}$ : fluorescence quantum yield

\begin{tabular}{lllll}
\hline Sample & Solvent & $\begin{array}{l}\lambda_{\text {abs }} \\
\max [\mathrm{nm}]\end{array}$ & $\begin{array}{l}\lambda_{\text {em }} \\
\max [\mathrm{nm}]\end{array}$ & $\Phi_{\mathrm{F}}$ \\
\hline \multirow{2}{*}{ BODIPY 3 } & $\mathrm{DMSO}^{a}$ & 626 & 634 & 0.80 \\
& Water $^{a}$ & 616 & 627 & - \\
fluo-CNOs & $\mathrm{DMSO}^{a}$ & 626 & 634 & 0.40
\end{tabular}

${ }^{a}$ Excitation at $550 \mathrm{~nm}$. Quantum yield: measured relative to zinc phthalocyanine. Quantum yield: 0.30 in $1 \%$ pyridine in toluene. ${ }^{43}$

fluo-CNOs have interesting fluorescence properties. Thus, their cyto-biocompatibility on live cells was tested to demonstrate their suitability as high-resolution cellular imaging probe with enhanced properties compare to previously reported ones. The in vitro toxic effects induced by oxi- and fluo-CNOs were evaluated on two different cells lines. The cell viability of MCF-7 (Fig. 4) and HeLa cells (Fig. S14 in the ESI†) exposed to different concentrations of CNOs $\left(0.5,1,5,10\right.$ and $\left.20 \mu \mathrm{g} \mathrm{mL}{ }^{-1}\right)$ for 12, 24, 48, and 72 hours was determined by means of colorimetric assay (WST1) (see ESI $\dagger$ for experimental details).

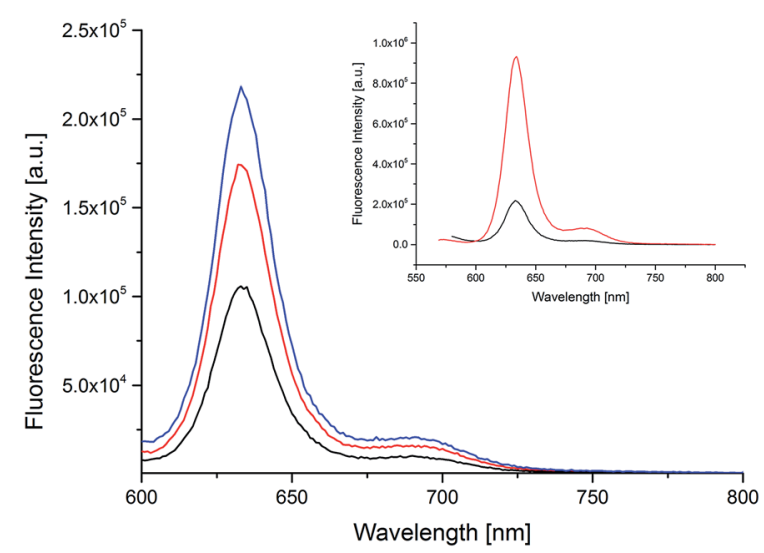

Fig. 3 Emission spectra in DMSO of fluo-CNOs at $5 \mu \mathrm{g} \mathrm{m}^{-1}$ (black line), $10 \mu \mathrm{g} \mathrm{mL}^{-1}$ (red line) and $20 \mu \mathrm{g} \mathrm{mL}^{-1}$ (blue line). Inset: emission spectra of BODIPY 3 (red line) and fluo-CNOs (black line) $\left(20 \mu \mathrm{g} \mathrm{m}^{-1}\right)$. Excitation at $550 \mathrm{~nm}$; emission at $634 \mathrm{~nm}$.
The results were expressed as percentage viability versus a control consisting of cells treated with the cell culture medium alone. As shown in Fig. $4 \mathrm{a}$ and Fig. S14a in the ESI, $\uparrow$ no cytotoxicity upon MCF7 and HeLa cells exposed to oxi-CNOs was observed, even at the higher concentration $\left(20 \mu \mathrm{g} \mathrm{mL} \mathrm{m}^{-1}\right)$ and after prolonged exposure (72 hours). With a similar behaviour, the cell viability of MCF7 and HeLa cells exposed to fluo-CNOs (Fig. $4 \mathrm{~b}$ and S14b in the ESI, $\uparrow$ respectively) was not affected, and it was higher than $80 \%$ for all the tested concentrations. Our results demonstrated the good cyto-biocompatibility of CNOs, encouraging their application as safe bioimaging probes.

\section{Cellular imaging}

Confocal live cell imaging was performed in order to track the intracellular fate of CNOs following their cell uptake and

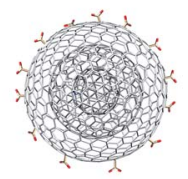

oxi- $\mathrm{CNOs}$

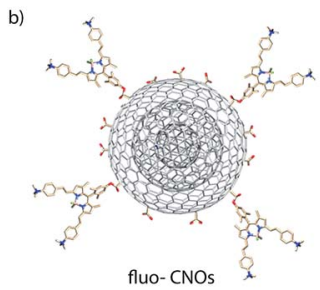

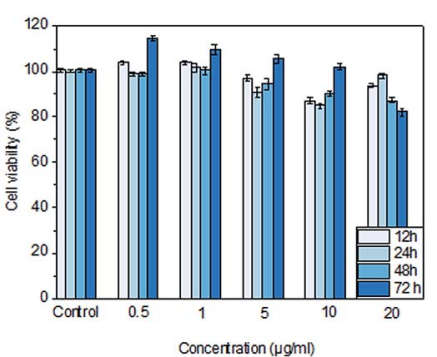

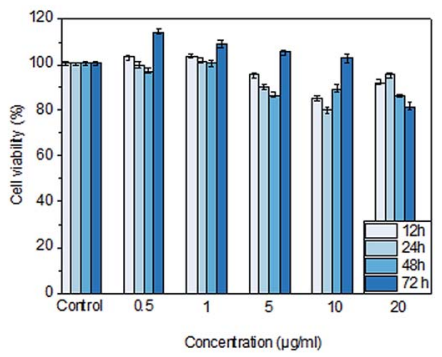

Fig. 4 Cellular viability of MCF7 cells treated with different concentrations $\left(0,51,5,10\right.$ and $20 \mu \mathrm{g} \mathrm{mL}^{-1}$ ) of oxi-CNOs (a) and fluo-CNOs (b) for 12, 24, 28 and 72 hours, revealed by WST 1 assay. Viability (\%) is evaluated for the samples of CNOs against a non-treated control. Data are expressed as mean \pm standard error as calculated from three separate experiments. 

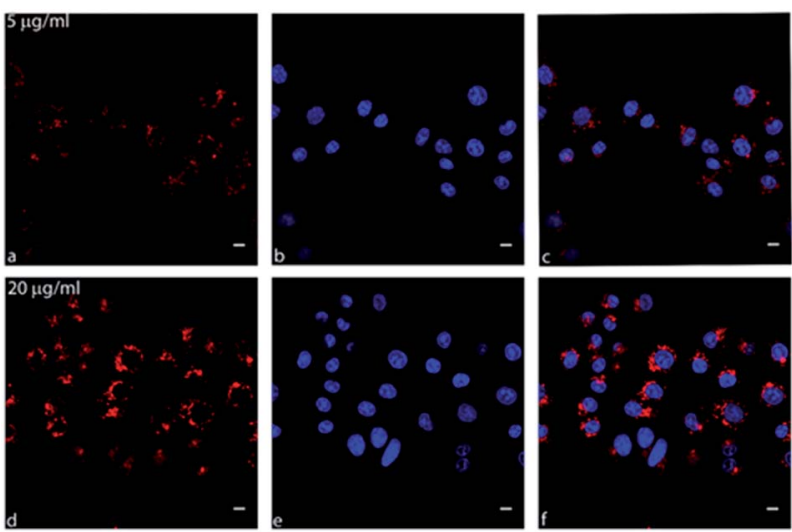

Fig. 5 Confocal images of living MCF7 cells after incubation for 24 hours with 5 and $20 \mu \mathrm{g} \mathrm{mL}^{-1}$ of fluo-CNOs. (a and d) fluo-CNOs (red); (b and e) nuclei stained with Hoechst 33342 (blue); (c and f) merged images. Scale bars $=10 \mu \mathrm{m}$.

characterize them as possible delivery platform. Fig. 5 show the internalization and localization of fluo-CNOs in living MCF cells, after incubation for 24 hours at concentration of 5 and $20 \mu \mathrm{g} \mathrm{mL} \mathrm{m}^{-1}$. More confocal images are shown in Fig. S15 in the ESI. $\dagger$ It could be clearly observed from the strong far-red fluorescence signal present in the cells (Fig. 5a and d), that fluoCNOs were successfully up-taken by MCF7 cells and were accumulated in cytoplasm around the nuclei (Fig. 5c and f). Fig. S16 in the ESI $\uparrow$ displays the cells volume rendering of the three dimensional confocal stacks, confirming the CNOs successful cell uptake and distribution throughout the cytosol. Moreover, we investigated the distribution of fluo-CNOs into vesicular compartments. Our previous in vitro studies demonstrated that green-labelled CNOs localized predominantly in the lysosomes. ${ }^{31}$

In order to confirm the distribution of fluo-CNOs in the cytoplasm, a lysosome specific dye, the LysoTracker Green (Fig. 6b and e), was used to identify the colocalization. As shown

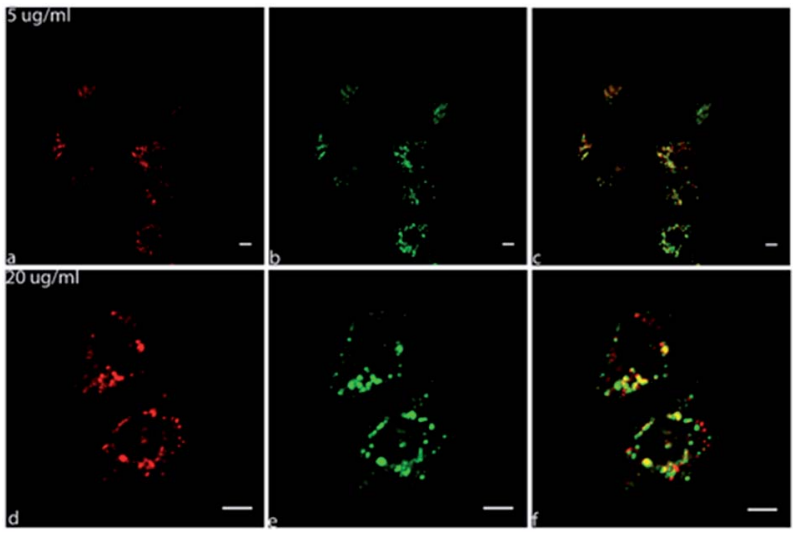

Fig. 6 Confocal images of MCF7 cells after incubation for 24 hours with 5 and $20 \mu \mathrm{g} \mathrm{mL}^{-1}$ of fluo CNOs. (a and d) fluo-CNOs (red); (b and e) lysosomes marked with Lysotracker green (green); (c and f) merged images. Scale bars $=10 \mu \mathrm{m}$. by the merged confocal images (Fig. 6c and f), the fluorescence of CNOs is in part overlapped with the Lysotracker green (yellow signal), demonstrating that fluo-CNOs deposited in the lysosomal vesicles. These data were confirmed by Fig. S17 in the ESI, $\dagger$ showing the cells volume rendering of the three dimensional confocal stacks. Furthermore, we evaluated the internalization of CNOs by colocalization analysis. Fig. S18B in the ESI $\dagger$ displays the colocalization mask (blue) applied to the corresponding confocal images of Fig. S18A in the ESI. $\dagger$ The value of Pearson's correlation coefficient (PCC), indicator of the degree of colocalization, was of 0.60987 . We demonstrated that fluo-CNOs were internalized extensively in MCF7 cell, with a partial localization in the lysosomes.

\section{Conclusions}

We reported on the development of a new generation of imaging probe with a high dispersibility in aqueous medium, high fluorescence intensity, cyto-biocompatibility and readily up-taken by cells. A water soluble red emitting BODIPY molecule with a high fluorescence quantum yield was designed for this purpose. The fluorescence intensity observed by cellular imaging was extraordinary even with a mild CNO surface functionalization with BODIPY 3. Thus we can envision a high degree of functionalization of fluo-CNOs with a therapeutic agent or a specific ligand for the creation of a targeted drug delivery system. No toxic behaviour was observed when the fluoCNOs were up-taken by HeLa and MCF7 cells up to 72 hours. Our findings demonstrated that fluo-CNOs are promising tools for high resolution imaging.

\section{Conflicts of interest}

There are no conflicts of interest to declare.

\section{Acknowledgements}

Istituto Italiano di Tecnologia is greatly acknowledged for funding. S. G. acknowledges the COST Action CA 15107 "MultiFunctional Nano-Carbon Composite Materials Network (MultiComp)". The authors wish to thank Dr Andrea Armirotti for Mass measurements (HRMS), Dr Luca Goldoni for the support on NMR measurements and IIT Nanochemistry and the Nanophysics departments for the facilities support.

\section{Notes and references}

1 S. Iijima, Nature, 1991, 354, 56-58.

2 P. M. Ajayan, Chem. Rev., 1999, 99, 1787-1800.

3 M. F. L. De Volder, S. H. Tawfick, R. H. Baughman and J. Hart, Science, 2013, 339, 535-539.

4 H. W. Kroto, J. R. Heath, S. C. O'Brien, R. F. Curl and R. E. Smalley, Nature, 1985, 318, 162-163.

5 S. Y. Lim, W. Shen and Z. Gao, Chem. Soc. Rev., 2014, 44, 362381.

6 J. Bartelmess and S. Giordani, Beilstein J. Nanotechnol., 2014, 5, 1980-1998. 
7 J. Key and J. F. Leary, Int. J. Nanomed., 2014, 9, 711-726.

8 K. Licha and C. Olbrich, Adv. Drug Delivery Rev., 2005, 57, 1087-1108.

9 C. Balas, Meas. Sci. Technol., 2009, 20, 104020.

10 R. Weissleder and V. Ntziachristos, Nat. Med., 2003, 9, 123128.

11 S. Giordani, J. Bartelmess, M. Frasconi, I. Biondi, S. Cheung, M. Grossi, D. Wu, L. Echegoyen and D. F. O'Shea, J. Mater. Chem. B, 2014, 2, 7459-7463.

12 J. Bartelmess, M. Baldrighi, V. Nardone, E. Parisini, D. Buck, L. Echegoyen and S. Giordani, Chem.-Eur. J., 2015, 21, 97279732.

13 D. Ugarte, Nature, 1992, 359, 707-709.

14 D. Ugarte, Carbon, 1995, 33, 989-993.

15 Y. Li, M. Kröger and W. K. Liu, Nanoscale, 2015, 7, 1663116646.

16 S. Iijima and J. Cryst, Growth, 1980, 50, 675-683.

17 N. Sano, H. Wang, M. Chhowalla, I. Alexandrou and G. A. Amaratunga, Nature, 2001, 414, 506-507.

18 S. K. Sonkar, M. Roy, D. G. Babar and S. Sarkar, Nanoscale, 2012, 4, 7670.

19 M. Ghosh, S. K. Sonkar, M. Saxena and S. Sarkar, Small, 2011, 7, 3170-3177.

20 X. H. Chen, F. M. Deng, J. X. Wang, H. S. Yang, G. T. Wu, X. B. Zhang, J. C. Peng and W. Z. Li, Chem. Phys. Lett., 2001, 336, 201-204.

21 L. Kuznetsov, A. L. Chuvilin, Y. V. Butenko, I. Y. Mal'kov and V. M. Titov, Chem. Phys. Lett., 1994, 222, 343-348.

22 A. Palkar, F. Melin, C. M. Cardona, B. Elliott, A. K. Naskar, D. D. Edie, A. Kumbhar and L. Echegoyen, Chem.-Asian J., 2007, 2, 625-633.

23 J. Chen, S. Z. Deng, J. Chen, Z. X. Yu and N. S. Xu, Appl. Phys. Lett., 1999, 74, 3651-3653.

24 C. A. Dyke and J. M. Tour, J. Phys. Chem. A, 2004, 108, 1115111159.

25 V. Georgakilas, K. Kordatos, M. Prato, D. M. Guldi, M. Holzinger and A. Hirsch, J. Am. Chem. Soc., 2002, 124, 760-761.

26 P. Singh, S. Campidelli, S. Giordani, D. Bonifazi, A. Bianco and M. Prato, Chem. Soc. Rev., 2009, 38, 2214.

27 A. Hirsch, Angew. Chem., Int. Ed., 2002, 41, 1853-1859.
28 Y.-L. Zhao and J. F. Stoddart, Acc. Chem. Res., 2009, 42, 11611171.

29 P. Petrov, F. Stassin, C. Pagnoulle and R. Jérôme, Chem. Commun., 2003, 23, 2904-2905.

30 K. E. Sapsford, W. R. Algar, L. Berti, K. B. Gemmill, B. J. Casey, E. Oh, M. H. Stewart and I. L. Medintz, Chem. Rev., 2013, 113, 1904-2074.

31 J. Bartelmess, M. Frasconi, P. B. Balakrishnan, A. Signorelli, L. Echegoyen, T. Pellegrino and S. Giordani, RSC Adv., 2015, 5, 50253-50258.

32 K. Flavin, I. Kopf, E. Del Canto, C. Navio, C. Bittencourt and S. Giordani, J. Mater. Chem., 2011, 21, 17881-17887.

33 M. Yang, K. Flavin, I. Kopf, G. Radics, C. H. A. Hearnden, G. J. McManus, B. Moran, A. Villalta-Cerdas, L. A. Echegoyen, S. Giordani and E. C. Lavelle, Small, 2013, 9, 4194-4206.

34 V. Georgakilas, D. M. Guldi, R. Signorini, R. Bozio and M. Prato, J. Am. Chem. Soc., 2003, 125, 14268-14269.

35 Y. Liu, R. L. Vander Wal and V. N. Khabashesku, Chem. Mater., 2007, 19, 778-786.

36 K. Flavin, M. N. Chaur, L. Echegoyen and S. Giordani, Org. Lett., 2010, 12, 840-843.

37 J. Bartelmess, E. De Luca, A. Signorelli, M. Baldrighi, M. Becce, R. Brescia, V. Nardone, E. Parisini, L. Echegoyen, P. P. Pompa and S. Giordani, Nanoscale, 2014, 6, 1376113769.

38 M. Frasconi, R. Marotta, L. Markey, K. Flavin, V. Spampinato, G. Ceccone, L. Echegoyen, E. M. Scanlan and S. Giordani, Chem.-Eur. J., 2015, 21, 19071-19080.

39 M. d'Amora, M. Rodio, J. Bartelmess, G. Sancataldo, R. Brescia, F. Cella Zanacchi, A. Diaspro and S. Giordani, Sci. Rep., 2016, 6, 1-9.

40 S. Çetindere, B. Çoşut, S. Yeşilot, M. Durmuş and A. Kiliç, Dyes Pigm., 2014, 101, 234-239.

41 E. Deniz, G. C. Isbasar, Ö. A. Bozdemir, L. T. Yildirim, A. Siemiarczuk and E. U. Akkaya, Org. Lett., 2008, 10, 34013403.

42 C. F. Chiu, N. Dementev and E. Borguet, J. Phys. Chem. A, 2011, 115, 9579-9584.

43 G. A. Crosby and J. N. Demas, Annu. Rev. Phys. Chem., 1971, 75, 991-1024. 